\title{
Presence and Absence of Outer Rings in Barred Galaxies
}

\author{
P. Rautiainen and H. Salo \\ Astronomy Division, Department of Physical Sciences, University of \\ Oulu, Finland
}

\section{Introduction}

Many barred spiral galaxies have an outer ring $(R)$ or a pseudoring $\left(R^{\prime}\right)$ in their outer disk. R. Buta (e.g. Buta 1995) has developed a detailed classification for different types of outer rings. Two main families of outer rings are recognized. In $R_{1}$ family the major axis of the ring is perpendicular to the bar and the spiral arms wind $180^{\circ}$ before meeting the other arm. In $R_{2}$ family the major axis of the ring is parallel to the bar and the spiral arms wind $270^{\circ}$.

The outer rings are usually considered to be related to the outer Lindblad resonance (OLR) of a rotating stellar bar. The ring shapes correspond to families of closed orbits in the vicinity of this resonance (e.g. Contopoulos \& Grosb $\varnothing l$ 1989 ), and also the sizes of the outer rings relative to the bar component fit to this suggestion. Furthermore, test-particle simulations which use analytical bar potentials can produce different types of outer rings near the OLR (Schwarz 1981; Byrd et al. 1994). We have obtained similar results when we modelled ringed galaxy IC 4214 by constructing the gravitational potential from near-IR observations (Buta et al. 1999; Salo et al. 1999).

However, this picture of resonance-related outer ring formation has some difficulties, like why there are many barred galaxies without an outer ring. There are at least three possibilities: 1) the time scale of the formation of the outer ring is so long that many barred galaxies have not yet developed one, 2) an interaction with another galaxy has destroyed the outer ring and 3) the pattern speed of the spiral arms deviates from that of the bar. The last possibility is supported by a simulation of Sellwood and Sparke (1988), where the pattern speed of the outer spiral arms was lower than that of the bar. However, if this is a common situation, it is hard to understand why the spiral arms often organize into two distinct outer ring families. We have studied the formation of different morphologies in the outer parts of barred galaxies by making $\mathrm{N}$-body simulations where the gas component is modelled by inelastically colliding test particles.

\section{Time scale of the outer ring formation}

In our N-body simulations, the nuclear rings can form almost immediately after the bar formation, and the inner ring in about 100 million years. On the other hand, it usually takes at least one gigayear from the bar formation before the outer pseudoring can appear in the gas component. This time scale is mainly dependent on the evolution of the stellar component. The effect of the collision frequency, i.e. the amount of dissipation, is surprisingly small. Thus systems 
with relatively young bars could lack outer rings, even if they have rings in their inner parts. Furthermore, in gas rich systems the bar induced gas inflow can lead to destruction of the bar in rather short time scale (e.g. Friedli \& Benz 1993; Norman et al. 1996). Therefore, some bars could be too short-lived to ever induce an outer ring. However, a large fraction of all spiral galaxies, about $2 / 3$, is composed of barred and intermediate types, suggesting that bars must be either long-lived or recurrent.

\section{Interactions}

Although it is possible that interactions trigger bar formation in at least some galaxies (Noguchi 1987, 1996), interactions can also destroy pre-existing structure. This could be the explanation for the absence of outer rings in some barred systems. Elmegreen et al. (1992) found that the fraction of outer rings decreases as the environment becomes richer. It is also possible that interaction converts outer rings into pseudorings. Elmegreen et al. also made simulations which support these suggestions.

\section{Multi-mode systems}

Even when the pattern speeds of the bar and the outer spiral arms are different, the structures can still be related by the non-linear mode coupling (Tagger et al. 1987; Masset \& Tagger 1997; Rautiainen \& Salo 1999): the resonances of the two modes overlap (e.g. corotation of the bar and the inner Lindblad resonance of the spiral) and energy and angular momentum can be exchanged between the modes. Whether the modes are independent or not, one would not expect outer ring formation to take place near the OLR of the bar when the pattern speeds are different.

We have studied the effect of multiple modes on outer ring formation by making N-body simulations with several disk-halo models and different values of Toomre-parameter $Q_{T}$. These models are two-dimensional and the halo is analytical. The general tendency is such that in the disk dominated systems the bar mode often dominates even beyond its OLR, whereas in the systems with a more pronounced halo component the slower modes may start to dominate beyond the corotation of the bar. In some models the domain of the bar corotating mode is larger in the gas component than in the stellar component. Increasing the initial value of $Q_{T}$ decreases the strengths of the modes, especially the spiral modes in the outer disk.

Surprisingly, the presence of multiple modes does not necessarily inhibit the formation of an outer ring, although in some cases the ring formation can be delayed by several gigayears. Of course, such a delay would contribute to the observed lack of outer rings. Furthermore, the clearest cases of outer rings are seen when the contribution of the mode corotating with the bar is strong near its OLR.

An interesting consequence of the presence of two modes with comparable strength near the OLR of the bar, is the almost cyclic evolution of some models, e.g. the one shown in Figure 1. The morphology of the outer structure changes between different outer pseudoring types and sometimes the outer ring even 

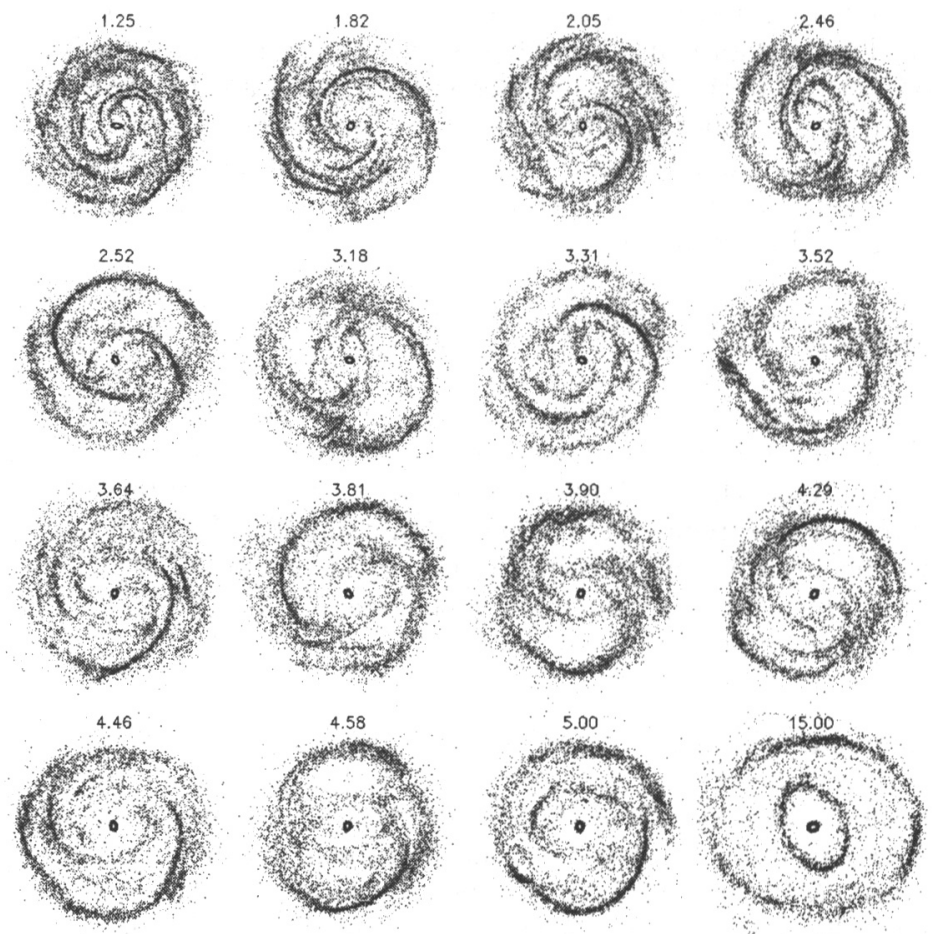

Figure 1. The evolution of the gas component in one of our simulations. Time is shown in gigayears. Several outer ring morphologies are presented during the evolution of the system: $R_{1}^{\prime}$ : e.g. $\mathrm{T}=2.46,4.29$, $R_{2}^{\prime}$, e.g. $\mathrm{T}=2.52,3.31, R_{1} R_{2}^{\prime}: \mathrm{T}=3.52,4.58$ and finally $R_{1}: \mathrm{T}=15.0$.

disappears. The time scale of these morphological changes is about 100 million years, whereas the orbital period near the OLR is considerably longer, about 500 million years. This cyclic behaviour is distinctly different from the simulations of Schwarz (1981), where the difference between the ring types was attributed to the initial gas particle distribution, or from Byrd et al. (1994), where the difference was related either with the bar pattern speed, or with the evolutionary change from type $R_{1}^{\prime}$ to type $R_{2}^{\prime}$.

Buta and Crocker (1991) found a correlation between the outer and inner structures: galaxies with $R_{1}$ or $R_{1}^{\prime}$ outer rings have more often inner rings, nuclear rings or dust lanes than galaxies with $R_{2}^{\prime}$ rings. This suggests that there is a profound difference between the galaxies showing different outer ring types, which contradicts with the above suggested cyclic evolution. However, Buta and Crocker's analysis was based on observations of only 22 galaxies, so it would be interesting to repeat the study with a larger galaxy sample.

In the model of Figure 1 the bar forms at about 1 Gyr after the start of the simulation and the first clearly classifiable outer ring is seen at about 2 Gyrs. At the same time, the pattern speed of the bar decreases by about $40 \%$. From 
$T=2$ Gyrs to $T=15$ Gyrs the bar pattern speed further decreases by $25 \%$, half of which happens during the first two gigayears. While this happens, the OLR moves outwards by about $30 \%$, which corresponds to the change in the size of the outer ring. This shows that constancy of the bar pattern speed is not necessary for the outer ring formation.

\section{Discussion}

There is a qualitative agreement between the results obtained by analytical bar models and N-body simulations: most outer rings are probably related to the outer Lindblad resonance of the bar component. However, the analytical bar models neglect the fact that the outer disk can play an active role, e.g. when there is more than one mode present in the disk. The N-body simulations show that the self gravitation of the outer disk can be an essential factor in the evolution of barred galaxies.

It seems that when several modes are present, the exact morphology of an individual galaxy can exhibit a cyclic change between different outer ring or pseudoring families, and the ring might even disappear occasionally. Some systems might lack the outer ring because the outer disk is dominated by a slower spiral mode or because the bar is so young that the outer ring has not had enough time to form. In interacting systems the outer parts can be so heavily disturbed that all signs of previous structure have disappeared.

\section{References}

Buta, R. 1995, ApJS, 96, 39

Buta, R., Crocker, D. A. 1991, AJ, 102, 1715

Buta, R., Purcell, G. B., Cobb, M. L., Crocker, D. A., Rautiainen, P., Salo, H. 1999, AJ, 117, 778

Byrd, G., Rautiainen, P., Salo, H., Buta, R., Crocker, D. A., 1994, AJ, 108, 476

Contopoulos, G., Grosbøl, P., 1989, A\&A Rev., 1, 261

Elmegreen, D. M., Elmegreen, B. G., Combes, F., Bellin, A. D. 1992, A\&A, 257, 17

Friedli, D., Benz, W., 1993, A\&A, 268, 65

Masset, F., Tagger, M. 1997, A\&A, 322, 442

Noguchi, M. 1987, MNRAS, 228, 635

Noguchi, M. 1996, ApJ, 469, 605

Norman, C. A., Sellwood, J. A., Hasan, H., 1996, ApJ, 462, 114

Rautiainen, P., Salo, H. 1999, accepted to A\&A

Salo, H., Rautiainen, P., Buta, R., Purcell, G. B., Lewis Cobb, M., Crocker,

D. A., Laurikainen, E. 1999, AJ, 117, 792

Schwarz, M. P., 1981, ApJ, 247, 77

Sellwood, J. A., Sparke, L. S. 1988, MNRAS, 231, 25

Tagger, M., Sygnet, J. F., Athanassoula, E., Pellat, R. 1987, ApJ, 318, L43 